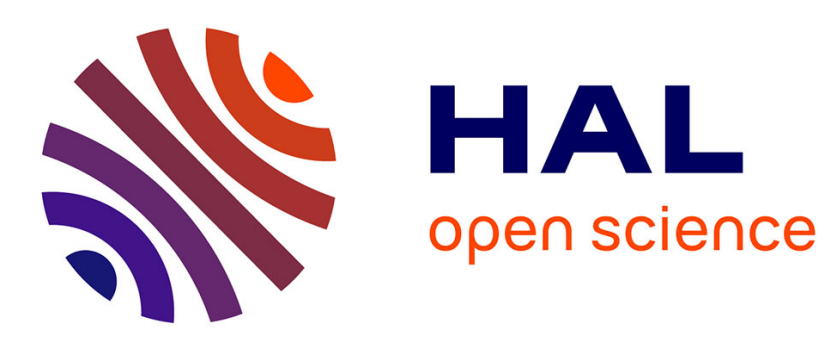

\title{
An Impact Test to Determine the Wave Speed in SHPB: Measurement and Uncertainty
}

Denis Brizard

\section{To cite this version:}

Denis Brizard. An Impact Test to Determine the Wave Speed in SHPB: Measurement and Uncertainty. Journal of Dynamic Behavior of Materials, 2020, 6, pp.45-52. 10.1007/s40870-019-00226-7 . hal02383273v2

\section{HAL Id: hal-02383273 \\ https://hal.science/hal-02383273v2}

Submitted on 2 Nov 2020

HAL is a multi-disciplinary open access archive for the deposit and dissemination of scientific research documents, whether they are published or not. The documents may come from teaching and research institutions in France or abroad, or from public or private research centers.
L'archive ouverte pluridisciplinaire HAL, est destinée au dépôt et à la diffusion de documents scientifiques de niveau recherche, publiés ou non, émanant des établissements d'enseignement et de recherche français ou étrangers, des laboratoires publics ou privés. 


\title{
An impact test to determine the wave speed in SHPB: measurement and uncertainty
}

\author{
D. Brizard ${ }^{1}$ \\ ${ }^{1}$ Univ Lyon, Université Claude Bernard Lyon 1, IFSTTAR, LBMC \\ UMR_T9406, F69622, Lyon, France \\ e-mail: denis.brizard@ifsttar.fr
}

\begin{abstract}
Post-processing the strain waves in Split Hopkinson Pressure Bars to get the stress-strain in the sample requires the knowledge of the characteristic wave speed $c_{0}$ in the measuring bars. In the context of metrology, the measurement uncertainty in the value of $c_{0}$ must be assessed. The aim is to minimize this uncertainty, which depends on the way $c_{0}$ is determined, as it has an impact on the uncertainty in the final stress and strain in the sample. The frequency domain method we introduce is based on an impact test on a single bar. The frequency spectrum of the impact response of the bar clearly exhibits the longitudinal resonant frequencies of the bar. The experimental dispersion curve is deduced from the spectrum and an optimization procedure was applied to determine the wave speed $c_{0}$ along with the Poisson's ratio and the uncertainty in the wave speed. This method gives a relative uncertainty in $c_{0}$ lower than $0.05 \%$ : it is mainly related to the uncertainty in the length of the bar (which is hard to reduce when using standard meter tape), which prevails over the uncertainty in the resonance frequencies of the bar. A precise value of the wave speed $c_{0}$ and of the associated measurement uncertainty is an important step in the context of Split Hopkinson pressure bars if we want to precisely assess the final uncertainty in Split Hopkinson pressure bars test results, which is at present scarcely done.
\end{abstract}

\section{Introduction}

Split Hopkinson pressure bars are commonly used to measure the stress-strain relationship of materials in the intermediate range of strain rate $\left(100 \mathrm{~s}^{-1}\right.$ to $\left.10000 \mathrm{~s}^{-1}\right)$ [1]. The main components of a SHPB device are a striker

Address(es) of author(s) should be given and two measuring bars [2]. The sample is placed between the two measuring bars and the striker hits the first measuring bar at a prescribed velocity. A strain wave propagates down the first measuring bar: part of the wave is reflected at the interface with the sample and part of the wave crosses the sample and propagates through the second measuring bar. Strain gauges are commonly used to measure the strain time series in each bar, they are often placed in the middle of the bars to avoid any overlay between the incident and reflected pulses. The stress and strain in the sample are computed from the incident, reflected and transmitted waves in the bars.

The value of the wave speed $c_{0}=\sqrt{E / \rho}$ is important in the post-processing of the SHPB tests because it appears in the equations of the strain rate and strain in the sample as 3 .

$$
\begin{aligned}
& \dot{\epsilon}_{s}(t)=\frac{-2 c_{0}}{l_{s}} \epsilon_{r}(t) \\
& \epsilon_{s}(t)=\frac{-2 c_{0}}{l_{s}} \int_{0}^{t} \epsilon_{r}(\tau) d \tau
\end{aligned}
$$

where $l_{s}$ is the initial length of the sample and $\epsilon_{r}(t)$ is the reflected wave measured in the input bar. $E$ is the modulus of elasticity and $\rho$ the density of the measuring bars.

In the context of quality in measurement, it is also necessary to determine the uncertainty in $c_{0}$ as it is involved in the final uncertainty in the stress and strain in the sample. The general approach to derive the measurement uncertainty in the stress and strain in the sample is presented in [4, however this paper does not detail how to assess the uncertainty in the wave speed. The present paper aims to fill this gap.

Naturally it is possible to compute $c_{0}$ from the measured values of $E$-on a tensile testing machine- and 
of $\rho$. However, the determination of the wave speed $c_{0}$ directly from a test made on SHPB is appealing, especially if other facilities are not available. The method described later in the present paper also allows the determination of Poisson's ratio $\nu$.

In the literature of SHPB, the accurate measurement of velocity $c_{0}$ is said to be critical [2] [5], however few details are given on how accurate it should be. In the case of low attenuation bars, the measurement methods may be classified into three categories.

The intuitive method of measuring the transit time between a pulse and its reflection seems rather simple but it faces some overlooked issues. The propagation of longitudinal waves in bars of finite diameter is dispersive [6], meaning that waves of different frequency $\omega$ have different phase velocity $c(\omega)$. This is described by the dispersion curve which gives the evolution of phase velocity with respect to either wave frequency or wave length (see Fig. 1 from [6] for instance); by definition $c_{0}=c(0)$. The shape of the pulse travelling down the bar is therefore affected: it is hard to determine the beginning of the pulse and to find the precise time shift between two pulses with slightly different shapes. Possibly noisy measurement can make this even harder. Furthermore, Davies [7] pointed out that "the mean velocity of propagation of the disturbance is decreased by dispersion", which means that measuring the velocity $c_{0}$ from a transit time procedure may be erroneous.

To overcome the change in shape of the pulse travelling down the bar, Lifshitz and Leber [8] introduced an iterative procedure to determine the velocity $c_{0}$ from an incident and a reflected pulse. One of the pulses was shifted -taking into account of dispersive effects- and compared to the other one, the value of $c_{0}$ was then adjusted "until the best fit is obtained". They however made the assumption that the low frequency part of the dispersion curve is insensitive to the value of $\nu$, and therefore did not discuss the determination of $\nu$.

The third method for the measurement of $c_{0}$ is based on vibration natural frequency, it was employed by Davies [7, Kolsky 9] and Zemanek and Rudnick [10. This method requires a specific device for the excitation of longitudinal resonances. Othman and Gary [11 proposed a method based on the analysis of the rod resonances from an impact test, however they only plot the experimental dispersion curve and do not deduce the values of $c_{0}$ and $\nu$, nor do they treat measurement uncertainties.

The scope of this paper is limited to bars in which the attenuation is low, typically metallic bars. On the contrary, attenuation cannot be neglected in polymeric bars. Bacon [12] proposed a non-parametric approach to obtain the experimental dispersion and attenuation curves of viscoelastic bars based on the transfer function between two observation points (or between an incident and a reflected pulse). Other bars identification procedures rather rely on parametric approaches: the aim is to determine a viscoelastic rheological model describing the behaviour of the material of the bars (see [13] for example).

In this article we introduce a parametric method for precisely determining the velocity $c_{0}$, along with Poisson's ratio $\nu$, from a simple impact test that can be done on the SHPB setup. The method uses the impact spectrum rather than the impact time series. Some specific points of the dispersion curve are deduced from the resonance frequencies in the impact spectrum and they feed an optimization procedure for finding the values of $c_{0}$ and $\nu$. Throughout all the process, measurement uncertainties are taken care of, in order to assess the final uncertainty in the wave speed.

This paper is organised as follows. The first part (Section 2) recalls the computation of the longitudinal resonant frequencies of a finite length rod. In Section 3 we derive the expression of the wave speed as function of resonance frequencies - used to plot the experimental dispersion curve--, compute the associated uncertainty and detail the optimization procedure to get the wave speed, the Poisson's ratio and the uncertainty in wave speed. Finally, in Section 4 the proposed method is applied on a practical example to illustrate its efficiency, it is compared to other methods and the influence of the uncertainty in $c_{0}$ on the SHPB stress-strain curve is illustrated.

\section{Longitudinal modes of a finite length rod}

Let $L$ be the bar lenght. Let $E, \rho$ and $\nu$ be respectively the Young modulus, the density and the Poisson's ratio of the bar.

\subsection{Resonance frequencies of $1 \mathrm{D} \operatorname{rod}$}

The wave equation of longitudinal waves in thin rods is [14] $(\operatorname{pp} 77,(2.1 .7)))$

$\frac{\partial^{2} u}{\partial t^{2}}+\frac{E}{\rho} \frac{\partial^{2} u}{\partial x^{2}}=0$

where $u(x, t)$ is the displacement at coordinate $x$ and time $t$. We can define the wave speed of the bar as $c_{0}=$ $\sqrt{E / \rho}$. Stationary solutions are of the form $u(x, t)=$ $U(t) \Phi(x)$ and Eq. 3 becomes

$\ddot{U} \Phi+c_{0}^{2} U \Phi "=0$ 
where the dot denotes the derivative with respect to the time variable $t$ and the prime denotes the derivative with respect to the space variable $x$. The previous equation can be reformulated as

$\frac{\ddot{U}}{U}=-c_{0}^{2} \frac{\Phi^{\prime \prime}}{\Phi}=-\omega^{2}, \quad \forall x, \forall t$

where $\omega$ is the circular frequency. The longitudinal modes are therefore the solutions of

$\Phi "-\frac{\omega^{2}}{c_{0}^{2}} \Phi=0$

Let $\lambda^{2}=\omega^{2} / c_{0}^{2}$. The solutions of Eq. 6 are of the form

$\Phi(x)=P \sin (\lambda x)+Q \cos (\lambda x)$

$\Phi^{\prime}(x)=\lambda P \cos (\lambda x)-\lambda Q \sin (\lambda x)$

where the constants $P$ and $Q$ are obtained from the boundary conditions, which are a null strain at $x=0$ and $x=L$

$\Phi^{\prime}(0)=\lambda P$

$\Phi^{\prime}(L)=-\lambda Q \sin (\lambda L)$

A null strain at the ends of the rod implies $P=0$ and as we are interested in non trivial solution (ie. $Q \neq 0$ ) Eq. 10 becomes $\sin (\lambda L)=0$. The longitudinal modes of the rod must therefore satisfy

$\lambda_{n}=\frac{n \pi}{L}, n \in \mathbb{N}$

The eigenfrequencies of the rod are

$f_{n}=\frac{n c_{0}}{2 L}, n \in \mathbb{N}$

\subsection{Antiresonances and observable resonances}

Whatever the position of the strain gauge on the bar, some vibration modes are not observable. These are the modes which have a vibration node located at the same position as the gauge. The resonant frequency is then as antiresonant frequency. In our case the strain gauge is located at the middle of the bar in order to minimize wave overlay, the additional condition for antiresonant frequency is therefore

$$
\Phi^{\prime}(L / 2)=0
$$

From Eq. 8 a non trivial solution still implies $\sin (\lambda L / 2)=$ 0 . The antiresonances therefore verify

$\lambda_{2 n}=\frac{2 n \pi}{L}, n \in \mathbb{N}$
The corresponding antiresonant frequencies are

$f_{2 n}=\frac{(2 n) c_{0}}{2 L}, n \in \mathbb{N}$

The observable resonant frequencies are deduced from Eq. 12 and Eq. 15 and if the strain gauge is in the middle of the bar we have

$f_{2 n+1}=\frac{(2 n+1) c_{0}}{2 L}, n \in \mathbb{N}$

3 Measurement of wave speed, Poisson's ratio and uncertainty in wave speed from the impact test

\subsection{Wave speed from impact test spectrum}

Since in reality the propagation of longitudinal waves in the measurement bars is dispersive [6] [7, we substitute $c_{0}$ by $c_{\exp }(f)$ in Eq. 16 . The observable resonant frequencies of the impact spectrum therefore give the wave speed at some specific frequencies

$c_{\exp }\left(f_{2 n+1}\right)=\frac{2 L f_{2 n+1}}{2 n+1}, n \in \mathbb{N}$

In the following, this is called the experimental dispersion curve (see Fig. 3b).

\subsection{Uncertainty in the value of the wave speed}

We use here the notations defined in the GUM (guide to the expression of measurement uncertainty [15]) and in the VIM (international vocabulary of metrology [16]). Let $u\left(x_{1}\right)$ denote the standard -in the sense of standard deviation- measurement uncertainty in $x_{1}$, it will be abbreviated as "uncertainty in $x_{1}$ ". The standard relative measurement uncertainty in $x_{1}$ is therefore $u\left(x_{1}\right) / x_{1}$, it will be abbreviated as "relative uncertainty in $x_{1}$ ".

The relative uncertainty in the computed velocity is deduced from Eq. 17 and the set of rules for functional relationships defined in [17]

$\left[\frac{u\left(c_{\exp }\right)}{c_{\exp }}\right]^{2}=\left[\frac{u(L)}{L}\right]^{2}+\left[\frac{u\left(f_{2 n+1}\right)}{f_{2 n+1}}\right]^{2}$

3.3 Optimization procedure on the experimental dispersion curve

The experimental dispersion curve (Eq. 17) -including measurement uncertainties (Eq. 18)- obtained from the impact test feeds an optimization procedure. The aim of the optimization is to find the values of the wave speed $c_{0}$, width of corridor $\Delta c_{0}$ and Poisson's ratio $\nu$ that 
best fit the experimental data including the uncertainty corridor, i.e. such that

$\operatorname{argmin} d_{\text {sup }}+d_{\text {mean }}+d_{\text {inf }}$

$c_{0}, \Delta c_{0}, \nu$

$d_{\text {mean }}=\operatorname{dist}\left(c\left(c_{0}, \nu\right), c_{\text {exp }}\right)$

$d_{\text {sup }}=\operatorname{dist}\left(c\left(c_{0}+\Delta c_{0}, \nu\right), c_{\exp }+u\left(c_{\exp }\right)\right)$

$d_{\text {inf }}=\operatorname{dist}\left(c\left(c_{0}-\Delta c_{0}, \nu\right), c_{\exp }-u\left(c_{\exp }\right)\right)$

where $c\left(c_{0}, \nu\right)$ is the computed dispersion curve whose parameters are wave speed $c_{0}$ and Poisson's ratio $\nu$. The computed dispersion curves are calculated by solving Pochhammer equation with a Newton algorithm (details are given in [18 and [19]). The distance dist $\left(c_{1}, c_{2}\right)$ is the average absolute difference between the two curves $c_{1}$ and $c_{2}$. The distance is evaluated at the experimental points and the average absolute difference is weighted with the inverse of the measurement uncertainty to avoid giving too much importance to the low frequency part of the experimental dispersion curve (see Fig. 3b).

The optimization algorithm is CMA-ES [20], we used the Python version available online.

The computed corridor for the dispersion curve depends on three parameters: $c_{0}, \Delta c_{0}$ and $\nu$ (see Fig. 3a). The lower limit of the corridor is $c\left(c_{0}-\Delta c_{0}, \nu\right)$ and the upper limit is $c\left(c_{0}+\Delta c_{0}, \nu\right)$. The value of $c_{0}$ only affects the vertical position of the dispersion curve without affecting its shape. The value of $\nu$ affects the shape of the velocity drop (see [6]).

No uncertainty in Poisson's ratio $\Delta \nu$ was used in the optimization procedure. Indeed, varying $\nu$ for a given value of $c_{0}$ only moves the high frequency part of the dispersion curve, the very low frequency part of the dispersion curve -before the drop in velocity- being insensitive to the value of $\nu$. So adding $\Delta \nu$ to the optimization procedure would not increase the fit of the experimental corridor -as it tends to widen the corridor as the frequency increases- nor would it give a value of $\Delta \nu$ other than zero. Indeed this was tested and the optimum $\Delta \nu$ was around $10^{-14}$.

\section{Application to $17-4 \mathrm{PH}$ steel bars}

\subsection{Impact test conditions}

The present method is applied on the specific SHPB device designed and built at our laboratory, LBMC UMR_T9406 (Iffstar/UCLB). Armco 17-4PH precipitation hardening steel is used for the measuring bars.

The diameter of the bars is $31.75 \mathrm{~mm}$, the bars were centerless ground to obtain a precise diameter, a good cylindricity and a low surface roughness. The end surfaces of the bars were machined on a lathe. The length of the bars is $L=3058 \mathrm{~mm}$, it is measured with a class II measuring tape. Directive 2014/32/EU [21] (Annex $\mathrm{X}$, Chapter 1) gives the maximum permissible error (MPE) between two non-consecutive scale marks as $A+B L_{r}+C$, where $A=0.3 \mathrm{~mm}, B=0.2 \mathrm{~mm} \mathrm{~m}^{-1}$, $C=0.2 \mathrm{~mm}$ and $L_{r}$ is the value of the length rounded to the next whole metre $\left(L_{r}=4 \mathrm{~m}\right.$ here). The uncertainty in the length is $u(L)=1.3 \mathrm{~mm}$, the relative uncertainty is $0.043 \%$.

The test for determining the wave speed $c_{0}$ is a single bar test: the strain wave is therefore reflected back and forth at the free ends of the bar. The high frequency content in the strain wave is ensured by the use of a short striker. The steel striker bar is $94.2 \mathrm{~mm}$ long and has a diameter of $30.0 \mathrm{~mm}$. The speed of the striker bar just before impact is $23 \mathrm{~m} \mathrm{~s}^{-1}$; yet this speed is not important for the determination of $c_{0}$ as changing the striker velocity has no influence on the wave duration.

Strain gauges are used to measure the wave travelling in the bar (4 gives the full details). However, the method presented in this article does not depend on any specific method of measurement of the waves propagating in the bars. For example Photon Doppler velocimetry, recently introduced for SHPB [22], could also be used.

The useful part of the signal is the one containing the multiple reflections of the wave in the bar (see Fig. 1). The duration of the time window is $T=191 \mathrm{~ms}$ and during this period the strain wave travels approximately 160 times the length $2 L$. The uncertainty in the measured frequency $u(f)$ is taken as the resolution in the frequency domain because the peaks are very sharp (see Fig 2), it is therefore directly linked to the period $T: u(f)=1 / T$.

4.2 Detection of the peaks in the frequency spectrum

The resonant frequencies are clearly visible in the frequency spectrum of the impact test (see Fig. 2). The peaks are automatically detected as the points with maximum amplitude between two antiresonances. The antiresonances are roughly determined using a first rough estimate of the wave speed $c_{0}$ and Eq. 15 Because the noise on the spectrum increases in high frequency, especially around antiresonances, the peak search zone is reduced to $60 \%$ of the frequency interval between two successive antiresonances (the light red bands in Fig. 2 are discarded). 


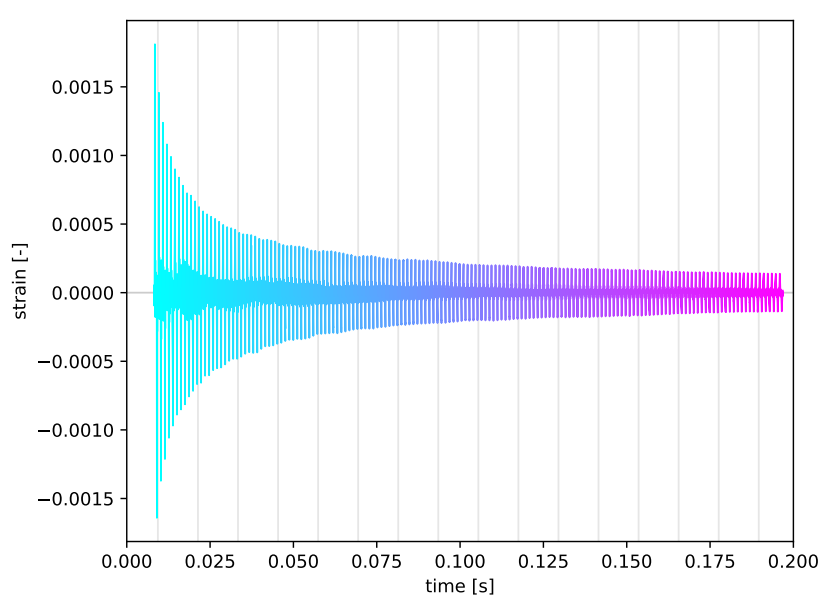

(a) Whole signal

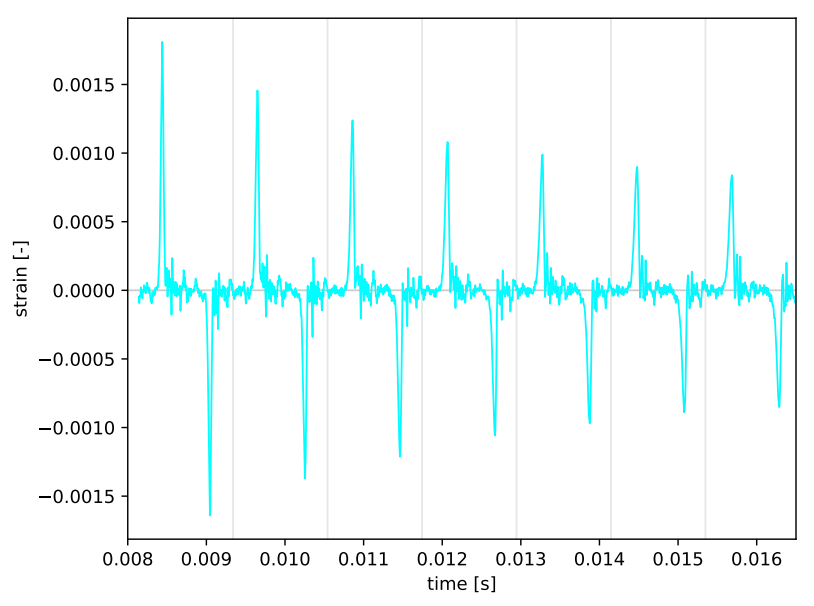

(b) First reflections

Fig. 1: Strain-time record for the impacted bar. Vertical lines $\mid$ indicate a travel of the wave over a distance of: (a) $10 \times 2 L,($ b) $2 L$.

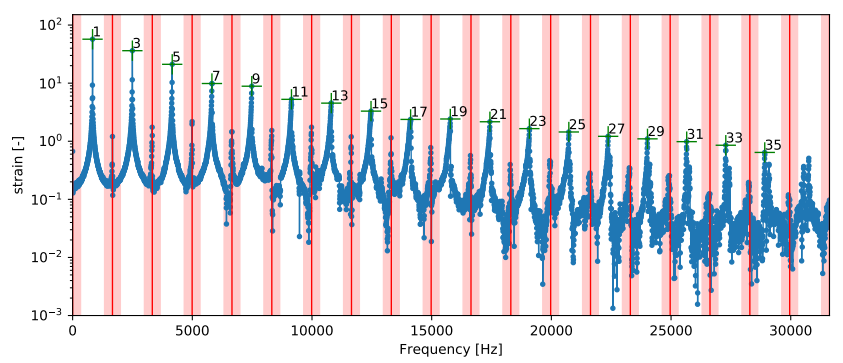

Fig. 2: Frequency spectrum of the impact test $(+$ : identified $f_{2 n+1}$ peaks with the corresponding value of $2 n+1 ; \mid$ : rough antiresonant frequencies $f_{2 n} ;$ : discarded peak search zone). Adapted from [23.
4.3 Wave speed and Poisson's ratio

Fig. 3b (blue curves) illustrates the experimental dispersion curve obtained from the post-processing of the impact spectrum with Eq.17. In fact, the impact test was repeated 5 times and all the corresponding experimental dispersion curves are displayed. These five tests give similar dispersion curves, except for the low frequency part where the discrepancy is noticeable.

The uncertainty in the velocity decreases as the frequency increases. This is due to the frequency term in Eq. $18 u\left(f_{2 n+1}\right)$ is constant and taken equal to the frequency resolution. The term $[u(f) / f]^{2}$ therefore decreases as the frequency increases. On the contrary, $[u(L) / L]^{2}$ is constant whatever the considered peak. From Eq. 18 it is clear that the uncertainty in $c$ tends asymptotically to the value of the uncertainty in $L$ as the frequency increases. The reduced uncertainty in the high frequency part of the curve is one of the interests of the present method.

The optimum dispersion curve and corridor is also plotted in Fig. 3b, the five tests were used in the optimization process. Practically, the five data sets were gathered as if it was only one dispersion curve. The agreement between the experimental curves and the optimum curve is very good. The optimization gives a wave speed of $5089.2 \mathrm{~m} \mathrm{~s}^{-1}$, a Poisson's ratio of 0.287 and corridor width of $\Delta c_{0}=2.46 \mathrm{~m} \mathrm{~s}^{-1}$. Not weighing the average for the distance criteria in the optimization gives the same values for $c_{0}$ and $\nu$, only $\Delta c_{0}$ is slightly higher and is equal to $2.7 \mathrm{~ms}^{-1}$.

By examining Fig. 3b we can see a close fit, in the high frequency part, between the experimental dispersion curve with measurement uncertainty and the optimum computed corridor: the uncertainty in the wave speed can be taken as the width of the corridor $u\left(c_{0}\right)=$ $\Delta c_{0}$. Taking $u\left(c_{0}\right)=2.5 \mathrm{~m} \mathrm{~s}^{-1}$ gives a relative uncertainty in the wave speed equal to $u\left(c_{0}\right) / c_{0}=0.05 \%$

In case a longer striker is used, the spectra are noisier and it is more difficult to automatically detect the resonant frequencies: dispersion curves are not as clean as with the shorter strikers. Indeed there are bumps on the experimental dispersion curve due to wrong peak picking in the impact spectrum. The optimization procedure using three impact tests ${ }^{1}$ performed with a $610 \mathrm{~mm}$ long striker gave the following values: $c_{0}=5089.8 \mathrm{~m} \mathrm{~s}^{-1}$, $\nu=0.290$ and $\Delta c_{0}=2.44 \mathrm{~m} \mathrm{~s}^{-1}$. The value for $c_{0}$ agrees with the one found with a short striker, concerning $\nu$ only the first two significant digit agree.

The proposed method does not allow to determine the uncertainty in the Poisson's ration $u(\nu)$. In SHPB tests, the Poisson's ratio is only required for the disper-

1 The number of resonance peaks is also 18 . 


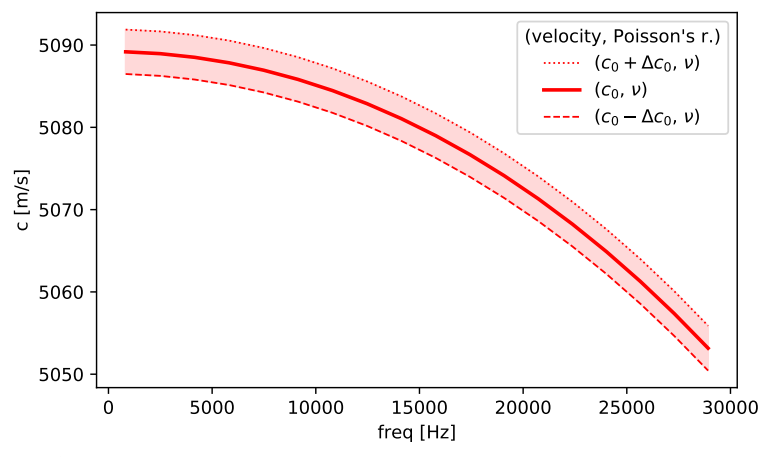

(a) Computed optimum dispersion curve with corridor

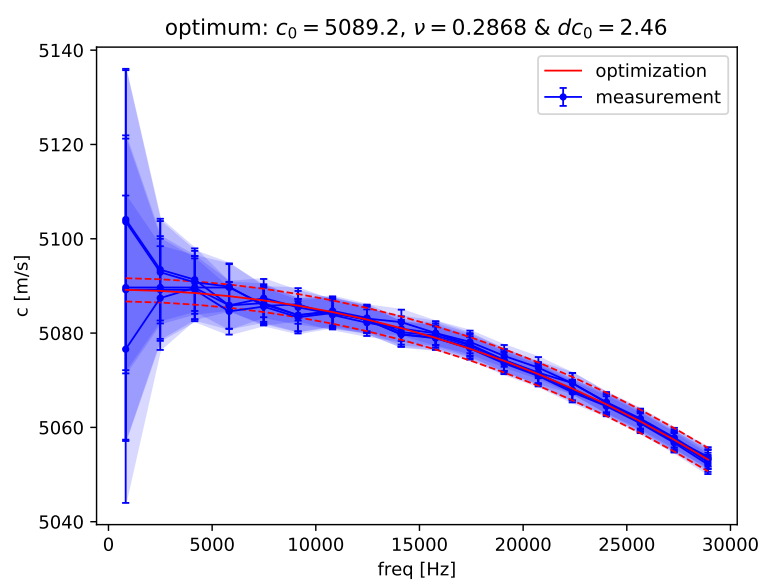

(b) Experimental dispersion curve and optmization result

Fig. 3: Computed and experimental dispersion curves

sion correction procedure of the raw pulses measured in the bars. For the uncertainty quantification of the whole SHPB post-processing (see [4]), the uncertainty in the dispersion curve may then be taken as the uncertainty in $c_{0}$ only. The uncertainty in $c_{0}$ is also required to quantify the uncertainty in the strain rate and in the strain from Eq. 1 and Eq. 2 So the undetermined value of the uncertainty in $\nu$ does not impede the assessment of the uncertainty in the stress and strain from SHPB tests.

Using only the first resonant frequency to determine the wave speed would lead to a much greater uncertainty in $c_{0}(0.6 \%$ in this case as opposed to $0.05 \%$ with the optimization procedure) and would not enable the determination of $\nu$.

\subsection{Comparison with other methods}

The material properties of $17-4 \mathrm{PH}$ steel in H1075 condition are given by the manufacturer (AKSteel) in two successive brochures. The 1994 brochure gives $\rho=7.81 \mathrm{~g} \mathrm{~cm}^{-3}$, $E=201000 \mathrm{MPa}$ and $\nu=0.291$, from which $c_{0}=$ $5073 \mathrm{~m} \mathrm{~s}^{-1}$. The 2018 brochure gives $\rho=7.81 \mathrm{~g} \mathrm{~cm}^{-3}$, $E=197 \times 10^{3} \mathrm{MPa}$ and $\nu=0.27$, from which $c_{0}=$ $5022 \mathrm{~m} \mathrm{~s}^{-1}$. No measurement uncertainties are given in the brochures, so it is hard to assess $u\left(c_{0}\right)$. The different values provided for $E$ and $\nu$ highlight the necessity to characterize the bar material and not to rely on the tabulated data.

Because of the effect of dispersion, the transit timemethod is not suitable to determine the value of $c_{0}$, be it by using the starting points of the wave or the peak of the wave. Both are affected by dispersion (see [14], §2.5.4 and §8.4.1). Fox and Curtis [24] however introduced a change in variable $\left(\left(t-x / c_{0}\right) / x^{1 / 3}\right.$, where $x$ is the propagation distance) which "should reduce the beginning portions of all strain-time records to a single curve". This was also used by Kaul and McCoy [25]. It is interesting to note that "the time of initial rise should vary inversely as the cube root of the distance of travel" [24]: that is why the starting points of the wave are not suitable for the transit time-method.

Instead of the transit time-method, we propose a modified time-method to determine $c_{0}$ from the straintime records. It consists in finding the optimal value of time shift $\Delta t_{\text {opt }}$ (within sampling accuracy of $0.2 \mu \mathrm{s}$ ) for which the waves best overlay in the modified time-plot, $c_{0}$ is then computed as $2 L / \Delta t_{\text {opt }}$. The best overlay is illustrated by Fig. 4 in which the full strain-time record of Fig. 1a was used (the record duration of nearly $0.2 \mathrm{~s}$ corresponds to a propagation distance of nearly $1 \mathrm{~km}$ ). The optimal value of $c_{0}$ is $5089.9 \mathrm{~m} \mathrm{~s}^{-1}$, it is in accordance with the value from the experimental dispersion curve of Section 4.3. We observed that a small variation of $\Delta t_{\text {opt }}$ of only a few samples clearly reduced the quality of the overlay of the pulses: that is why the value of $c_{0}$ can reasonably be given with 5 significant figures. However, with this transit time-method it is difficult to assess the uncertainty in $c_{0}$ and the value of $\nu$ cannot be determined.

\subsection{Uncertainty corridor on the stress-strain curve} with SHPB

In order to illustrate the influence of the uncertainty in the wave speed, the uncertainty corridor on the stressstrain curve of a typical SHPB test is computed for two values of $u\left(c_{0}\right) / c_{0}$, namely $0.6 \%$ and $0.05 \%$.

We assume that the modulus of elasticity is computed from the wave speed and the density as $E=\rho c_{0}^{2}$. The following relationship holds for the uncertainty in 


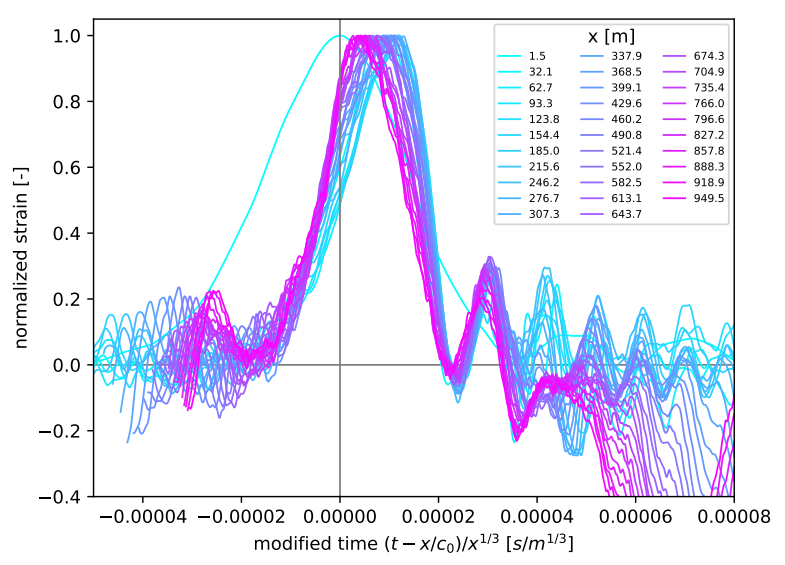

Fig. 4: Normalized strain with respect to modified time (according to [24]). For sake of clarity, only one wave (pulse propagating on a distance of $2 L$ ) out of 5 is plotted, and the abscissa is restrained to the compression part of the strain wave.

$E($ see [17])

$\left[\frac{u(E)}{E}\right]^{2}=\left[\frac{u(\rho)}{\rho}\right]^{2}+4\left[\frac{u\left(c_{0}\right)}{c_{0}}\right]^{2}$

The value of the density given by the manufacturer is $\rho=7.81 \mathrm{~g} \mathrm{~cm}^{-3}$. Following [26] (Rule 1, page 23), we can assume that the uncertainty in $\rho$ is on its last significant digit: $u(\rho)=0.005$. This gives $u(\rho) / \rho=0.06 \%$.

In case $u\left(c_{0}\right) / c_{0}=0.6 \%$, then $u(E) / E=1.2 \%$. In case $u\left(c_{0}\right) / c_{0}=0.05 \%$, then $u(E) / E=0.12 \%$. Both cases are used to compute the uncertainty corridor around the stress-strain curve of a copper sample tested with SHPB. All the details of the method to get the uncertainty corridor are given in [4]. The influence of $u\left(c_{0}\right)$ on the width of the uncertainty corridor is noticeable (see Fig. 5 ).

The importance of the uncertainty corridor is quantified by the ratio of the area of the uncertainty corridor upon the area of the stress-strain curve. The stressstrain curve contour is closed by a point located on the $\mathrm{x}$-axis at the vertical of the last point of the stress strain curve. This ratio is equal to $10.4 \%$ when $u\left(c_{0}\right) / c_{0}=$ $0.6 \%$ and $3.5 \%$ when $u\left(c_{0}\right) / c_{0}=0.05 \%$ : the area of the uncertainty corridor is divided by 3 when the uncertainty in $c_{0}$ is divided by 10 .

\section{Conclusion}

This article introduces a method for the determination of the wave speed $c_{0}$, uncertainty in wave speed $u\left(c_{0}\right)$ and Poisson's ratio $\nu$ of SHPB measurement bars. This

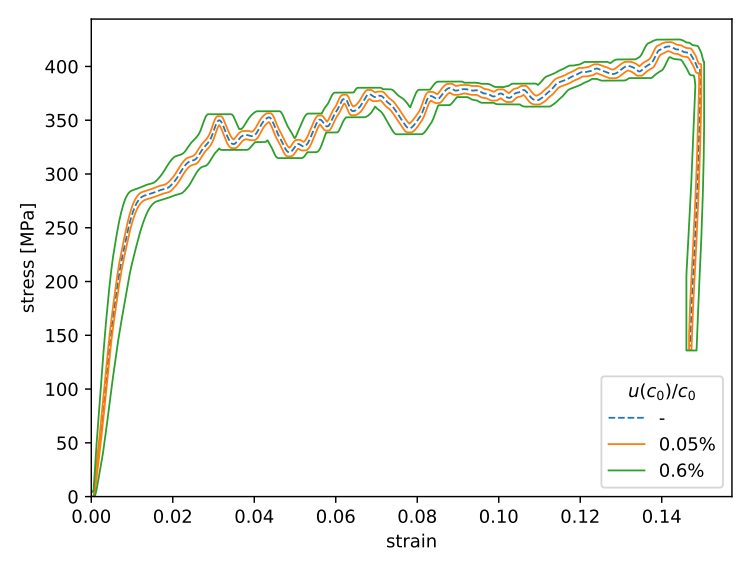

Fig. 5: Influence of the relative uncertainty in $c_{0}$ on the stress-strain curve in the sample with SHPB (copper sample, signals are low-pass filtered at $200 \mathrm{kHz}$ )

method is based on a straightforward impact test on a single bar with a short striker, which is an easy experiment to perform on a SHPB device. From the impact test spectrum, the sharp peaks that correspond to the resonant frequencies of the bar are easily identified. These resonances are used to obtain some specific points of the experimental dispersion curve. Finally, the points of the experimental dispersion curve feed a simple optimization procedure which outputs the values of $c_{0}, u\left(c_{0}\right)$ and $\nu$.

With this method, the relative uncertainty in $c_{0}$ is of the order of magnitude of the relative uncertainty in the bar length, ie. around $0.05 \%$, because the uncertainty in the frequency is very small.

In the context of quality in measurement with SHPB, this is an important preliminary step required to assess the uncertainty in the strain and strain rate in the sample 4].

\section{Acknowledgements}

The author is grateful to Pr Eric Jacquelin and Pr Sylvie Ronel for their help and suggestions of improvement.

\section{References}

1. Howard Kuhn. ASM Handbook: Volume 8: Mechanical Testing and Evaluation, volume 8. ASM International, 10 edition, September 2000.

2. Bazle A Gama, Sergey L Lopatnikov, and John W Gillespie. Hopkinson bar experimental technique: A critical review. Applied Mechanics Reviews, 57(4):223, 2004. 
3. Zhouhua Li and John Lambros. Determination of the dynamic response of brittle composites by the use of the split Hopkinson pressure bar. Composites Science and Technology, 59(7):1097-1107, May 1999.

4. D. Brizard, S. Ronel, and E. Jacquelin. Estimating Measurement Uncertainty on Stress-Strain Curves from SHPB. Experimental Mechanics, 57(5):735-742, June 2017.

5. George T. Gray III. Classic split-Hopkinson pressure bar testing. ASM Handbook, Mechanical testing and evaluation, 8:462-476, 2000.

6. Dennison Bancroft. The Velocity of Longitudinal Waves in Cylindrical Bars. Physical Review, 59(7):588-593, 1941.

7. R. M. Davies. A Critical Study of the Hopkinson Pressure Bar. Philosophical Transactions of the Royal Society of London A: Mathematical, Physical and Engineering Sciences, 240(821):375-457, January 1948.

8. J. M. Lifshitz and H. Leber. Data processing in the split Hopkinson pressure bar tests. International Journal of Impact Engineering, 15(6):723-733, 1994.

9. H. Kolsky. An Investigation of the Mechanical Properties of Materials at very High Rates of Loading. Proceedings of the Physical Society. Section B, 62(11):676-700, November 1949.

10. Joseph Zemanek and Isadore Rudnick. Attenuation and Dispersion of Elastic Waves in a Cylindrical Bar. The Journal of the Acoustical Society of America, 33(10):1283-1288, October 1961.

11. R. Othman, G. Gary, R. H. Blanc, M.n. Bussac, and P. Collet. Dispersion identification using the fourier analysis of resonances in elastic and viscoelastic rods. In Acoustics, Mechanics, and the Related Topics of Mathematical Analysis, pages 229-235. World Scientific, January 2003.

12. C. Bacon. An experimental method for considering dispersion and attenuation in a viscoelastic Hopkinson bar. Experimental Mechanics, 38(4):242-249, December 1998.

13. H. S. U. Butt, P. Xue, T. Z Jiang, and B. Wang. Parametric identification for material of viscoelastic SHPB from wave propagation data incorporating geometrical effects. International Journal of Mechanical Sciences, 91:46-54, 2015.

14. Karl F. Graff. Wave Motion in Elastic Solids. Dover Publications, June 1991.

15. Evaluation of measurement data - Guide to the expression of uncertainty in measurement. JCGM 100: 2008 (GUM 1995 with minor corrections), BIPM Joint Committee for Guides in Metrology, Paris, Sèvres, 2008.

16. International vocabulary of metrology - Basic and general concepts and associated terms, 3rd edn. JCGM 200: 2008 (VIM), BIPM Joint Committee for Guides in Metrology, Paris, Sèvres, 2008.

17. Ian Farrance and Robert Frenkel. Uncertainty of Measurement: A Review of the Rules for Calculating Uncertainty Components through Functional Relationships. The Clinical Biochemist Reviews, 33(2):49-75, May 2012.

18. D. Brizard, E. Jacquelin, and S. Ronel. Polynomial mode approximation for longitudinal wave dispersion in circular rods. Journal of Sound and Vibration, 439:388-397, January 2019.

19. D. Brizard and E. Jacquelin. Uncertainty quantification and global sensitivity analysis of longitudinal wave propagation in circular bars. Application to SHPB device. International Journal of Solids and Structures, 134:264-271, March 2018.

20. Nikolaus Hansen. The CMA Evolution Strategy: A Tutorial. arXiv:1604.00772 [cs, stat], April 2016. arXiv: 1604.00772.

21. Directive 2014/32/EU of the European Parliament and of the Council of 26 February 2014 on the harmonisation of the laws of the Member States relating to the making available on the market of measuring instruments (recast), February 2014.

22. C. Avinadav, Y. Ashuach, and R. Kreif. Interferometrybased Kolsky bar apparatus. Review of Scientific Instruments, 82(7):073908, July 2011.

23. Denis Brizard. Experimental measurement of value and associated uncertainty of wave speed in Hopkinson bars with an accurate and robust method. In International Conference on Uncertainty in Structural Dynamics USD, Leuven, Belgium, September 2018.

24. George Fox and C. W. Curtis. Elastic Strain Produced by Sudden Application of Pressure to One End of a Cylindrical Bar. II. Experimental Observations. The Journal of the Acoustical Society of America, 30(6):559-563, 1958.

25. R. K. Kaul and J. J. McCoy. Propagation of Axisymmetric Waves in a Circular Semiinfinite Elastic Rod. The Journal of the Acoustical Society of America, 36(4):653-660, April 1964.

26. Les Kirkup and Bob Frenkel. An Introduction to Uncertainty in Measurement: Using the GUM (Guide to the Expression of Uncertainty in Measurement). Cambridge Universtity Press, Cambridge, 2006. 